\title{
Individual bottle brush molecules in dense 2D layers restoring high degree of extension after collapse-decollapse cycle: Directly measured scaling exponent
}

\author{
M.O. Gallyamov ${ }^{1, a}$, B. Tartsch ${ }^{2}$, I.I. Potemkin ${ }^{1,3, b}$, H.G. Börner ${ }^{4,5}$, K. Matyjaszewski ${ }^{4}$, A.R. Khokhlov ${ }^{1,3}$, and \\ M. Möller6 \\ 1 Faculty of Physics, M. V. Lomonosov Moscow State University, Leninskie gory 1-2, GSP-1, 119991 Moscow, Russia \\ 2 Organische Chemie III, Universität Ulm, Albert-Einstein-Allee 11, D-89069 Ulm, Germany \\ ${ }^{3}$ Institute of Polymer Science, Universität Ulm, Albert-Einstein-Allee 47, D-89069 Ulm, Germany \\ 4 Department of Chemistry, Mellon Institute, Carnegie Mellon University, 4400 Fifth Avenue, PA 15213 Pittsburgh, USA \\ ${ }^{5}$ Department of Colloid Chemistry, Max Planck Institute of Colloids and Interfaces, D-14424 Potsdam-Golm, Germany \\ ${ }^{6}$ Institute of Technical and Macromolecular Chemistry, RWTH Aachen and DWI at the RWTH Aachen e.V., Pauwelsstr. 8, \\ D-52056 Aachen, Germany
}

Received 27 November 2008 and Received in final form 2 February 2009

Published online: 6 May 2009 - C EDP Sciences / Società Italiana di Fisica / Springer-Verlag 2009

\begin{abstract}
We prepared dense films of adsorbed brush-like macromolecules on mica substrate by transfer of compressed Langmuir monolayers from water subphase. The main macromolecular contours in the dense films were clearly resolved by SFM. The films were subjected to successive treatments by ethanol and water vapours. In accordance with previous results for isolated macromolecules, the films underwent collapse and subsequent decollapse morphological transformations in the changing vapour environment. Statistical analysis of the macromolecular dimensions in the films allowed us to measure the values of the scaling exponent $\nu$ determining the correlation between mean lateral and linear dimensions of the macromolecules. The analysis showed that the macromolecular conformations in the film as transferred were similar to the previously described conformations of the same macromolecules deposited directly on mica as isolated chains at much lower surface densities. The determined $\nu$ was close to the 0.75 value corresponding to the 2D SAW statistics. We assumed that the molecules retained the high degree of extension during the compression step due to suppressed reorganisation of the side chains. Differently from previous observations for isolated macromolecules, the restored conformations in the dense films after collapse-decollapse cycle were more extended with the $\nu$ of about 0.73 value. A theoretical explanation of the high degree of re-extension is proposed.
\end{abstract}

PACS. 36.20.Ey Conformation (statistics and dynamics) - 68.47.Pe Langmuir-Blodgett films on solids; polymers on surfaces; biological molecules on surfaces - 82.37.-j Single molecule kinetics - 82.37.Gk STM and AFM manipulations of a single molecule

\section{Introduction}

There are many different parameters to describe quantitatively the conformation of an adsorbed polymer chain. Among the general ones, one can mention the scaling exponent $\nu$. It describes how a mean lateral macromolecular size (e.g., mean-square end-to-end distance) scales with linear size (i.e. degree of polymerization, contour length) of polymer chains. For different two-dimensional (2D) sys-

\footnotetext{
a e-mail: glm@spm.phys.msu.ru

b e-mail: igor@polly.phys.msu.ru
}

tems the scaling exponent $\nu$ is described theoretically [1], and it is also a subject of numerous experimental verifications. Two main approaches are usually explored to determine the 2D scaling exponent $\nu$ experimentally: first, by indirect analysis of rheological properties of Langmuir monolayers (response of the whole ensemble to an external stress); second, by direct microscopic determination of macromolecular dimensions (tracing directly visualized macromolecular contours). The majority of the results are obtained for the systems with isolated single chains.

Within the framework of the first approach there were several reports on the $2 \mathrm{D}$ scaling exponent $\nu$ values deter- 
mined for polymeric Langmuir films. The most exploited method is based on linear fitting the dependence of surface pressure on surface concentration in semidilute regime. Thus the following scaling exponents were reported for different polymers on aqueous subphases: 0.79 [2] and 0.78 [3] for poly(vinyl acetate), 0.53 [4] and 0.56 [2] for poly(methyl methacrylate), 0.78 [4], 0.77 and $0.51[5,6]$ for poly(methyl acrylate) at different temperatures, 0.52 for poly(4-hydroxystyrene) [7], 0.53 for poly(tert-butyl methacrylate) [8]. The values of $0.77-0.79$ were attributed to the well-known $0.75(3 / 4)$ exact scaling exponent for $2 \mathrm{D}$ self-avoiding walk (SAW) statistics [9]. This scaling is indicative of an extended 2D polymer coil with excludedvolume interactions: without intersections. The lower values $0.52-0.56$ were mostly attributed to a $\theta$-point scaling exponent. But they are also close to the reference 0.5 value: for example, in $2 \mathrm{D}$ case a compacted $2 \mathrm{D}$ (flat) globule has such a statistics.

Within the framework of the second approach several different direct microscopic techniques were applied: transmission electron microscopy (TEM), fluorescence microscopy (FM) and scanning force microscopy (SFM). The experimental scheme and image analysis for determination of the scaling exponent using TEM were well established already quite a long time ago. The procedure was based on direct tracing of visualized macromolecular profiles and was mostly applied for an analysis of biopolymer macromolecules. Indeed they are rather big and thus easy to be visualized by TEM, especially when the proper shadowing or contrasting procedure is applied. It was proved experimentally that for DNA molecules there were two typical values of the scaling exponent to be measured, depending on the degree of irreversibility of the macromolecular adsorption during sample preparation [10]. A glow-discharge deposition technique resulted in strong adsorption of DNA molecules on the positively charged carbon surface. Therefore the corresponding scaling exponents were about 0.59 [10]. These scaling exponents coincided well with the exact value for 3D SAW statistics. Thus, such values were attributed to a surface-trapped conformation of a $2 \mathrm{D}$ projection of the $3 \mathrm{D}$ coil. This is not really a $2 \mathrm{D}$ conformation, as far as the molecules deposited in such manner have a lot of intersections. Indeed the intersections are forbidden or suppressed in a strict 2D case. When DNA molecules during deposition were incorporated in a protein film (of cytochrome c), they retained more freedom to reorganize. Therefore, the cytochrome c deposition technique resulted in a more relaxed conformation with the scaling exponents of about 0.72 [10], that is close to the 2D SAW statistics values [10]. For some other biopolymer deposited on mica the scaling exponents were determined and reported also: 0.57 for xanthan Kelzan, 0.63 for alginate, 0.68 for xanthan PX061, 0.69 for scleroglucan, 0.73 for xylinan [11].

Following the same scheme, the FM was explored to monitor a conformation of DNA molecules on a mobile cationic lipid membrane interface $[12,13]$. As far as the observed DNA molecules kept a possibility to rearrange freely in $2 \mathrm{D}$, the obtained 0.79 scaling exponent value was close to the expected 2D SAW statistics value. Some- what lower values of 0.65 and 0.68 were obtained for DNA molecules partially confined in a $110 \mathrm{~nm}$ slit [14]. The reason of the lower values was that the conformation of the partially confined molecules was not really a $2 \mathrm{D}$ one, but rather intermediate of the $2 \mathrm{D}$ and $3 \mathrm{D}$ ones.

Recent SFM experiments usually follow the same paradigm, which was developed previously for the TEM and FM methods. Thus, for surface-trapped DNA molecules on APTES-modified mica the 0.59 value was observed [15], as expected for a projection of a 3D coil. In the next experiment the 0.59 value was confirmed for kinetically trapped 3D configuration on APTES-modified mica and the intermediate 0.67 value for weaker adsorption on $\mathrm{Mg}^{2+}$ modified mica was reported [16]. Any intermediate values may be expected for an ensemble of partially relaxed molecules, which is to be described thus as a mixed state.

High spatial resolution of SFM allowed researches to study not only persistent biopolymers, but flexible synthetic polymer chains as well. Thus the 0.56 scaling exponent value was obtained for poly(4-vinylpyridine) (P4VP) single chains surface-trapped by mica [17]. Though, at the same conditions of deposition, larger values of $0.7-0.8$ were obtained for $\mathrm{P} 4 \mathrm{VP}$ salts with hydrophobic pendant groups and for their complexes with surfactants introducing additional hydrophobic side chains [17]. Such scaling exponents were close to the 2D SAW value, hence, the hydrophobically modified P4VP molecules were able to rearrange more freely during the adsorption because of the reduced attraction to mica surface. The also close 0.76 value was reported for P2VP chains as adsorbed on mica from solutions at low $p \mathrm{H}$ and at high ionic strength [18], i.e. at the conditions also favourable for rearrangement during adsorption (lower electrostatic attraction). Another close value of 0.73 was found for the relaxed linear P2VP molecules on mica after their equilibration by an exposure to water vapour [19].

There was another interesting and rather peculiar method proposed to determine the scaling exponent with the help of SFM [20]. Individual polysaccharide chains were randomly picked up by the SFM tip. According to the subsequent analysis of a set of the recorded force-distance curves the scaling exponent of 0.74 was found for carboxymethyl-amylose chains pinned to a glass slide [20].

The above-described experimental results were obtained for isolated polymer chains in diluted or semidiluted regimes at low surface concentrations. In such conditions the adsorbed molecules are non-contacting or only weekly contacting. The interpretation of the accumulated experimental results for the diluted systems is mostly quite straightforward in the framework of existing theoretical conceptions.

But it seems to be more intriguing to study the conformation of macromolecules inside denser films. Indeed, on the one hand, there are several contradicting theoretical models of the polymer chain behaviour in such dense monolayers. The models predict different macromolecular behaviour in dense films regarding the following aspects: whether the polymer chains are segregated or interpenetrating, whether they are compacted or extended and what is the scaling exponent $\nu$ value. 
Thus, the 0.5 value of the $2 \mathrm{D} \nu$ scaling exponent was theoretically predicted for self-avoiding chains (no intersections) in dense 2D monolayers [21]. The somehow higher value of $0.5+\varepsilon$ ( $\varepsilon$ due to a logarithmic correction) was calculated theoretically for a model with allowed intersections [22]. Here strong interpenetration of the polymer chains was predicted [22]. In a recent paper [23] two types of dense polymers in $2 \mathrm{D}$ were theoretically considered: 1) when chain intersections were totally forbidden, and 2) when they were allowed but to some extent (suppressed by an energetic penalty). Quite different results were obtained for the two models. According to model 1), the polymer chains were compacted (crumpled) and segregated, whereas the scaling exponent was equal to 0.5 [23]. According to model 2) the chains were strongly overlapping and entangled, whereas the scaling exponent was somewhat higher: $0.5+\varepsilon$ (a logarithmic correction) [23].

Extensive computer simulations did not clarify the picture: the results here obtained were also rather scattered. Thus, as a result of Monte Carlo simulations for polydisperse melt, $0.54-0.55$ (and up to 0.58 ) values were obtained [24]. Here the chains were reported also to be interpenetrating [24]. Another simulation depicted completely segregated chains with the exponent of 0.5 [25]. Still another simulating approach gave $0.52-0.54$ scaling exponent values [26]. For a quasi-2D melt (intersections were possible, but suppressed) partially segregated chains with the 0.5 scaling exponent were simulated [27]. On the other hand, considerable interpenetration between polymer molecules and the 0.54 scaling exponent were also reported, as a result of yet another simulating scheme [28].

Direct experimental verifications of the proposed models are strongly desirable. But the experimental results on dense films are not numerous by now. They were mostly obtained by direct microscopic methods. The serious obstacle here is that it is sometimes rather tricky to distinguish the conformation of single chains inside dense films. Thus, brush-like copolymers with poly(methyl methacrylate) (PMMA) backbones and short poly(ethylene oxide) (PEO) side chains were directly observed at the surface of the ultrathin film of the same polymer by TEM [29]. In order to visualize only those molecules, which were indeed located at the interface (2D), partial labeling by gold nanoparticles was applied. Only nanoparticles continuously connected in a chain-like manner were ascribed to single-polymer macromolecules at the surface and were further taken into account for a numerical analysis. As a result, the experimental value of 0.69 was reported for the scaling exponent $\nu$ describing the statistics of the macromolecular profiles (backbones) [29].

Scanning near-field optical microscopy was applied to study conformation of single dye-labelled poly(isobutylmethacrylate) chains in dense Langmuir-Blodgett monolayers [30]. If the polymer chains in a homogeneous monolayer were surrounded by the same molecules (i.e. of the same polymerisation degree), then the contracted pancake-like conformation with scaling exponent of 0.5 was reported. On the contrary, the labelled polymer chains were significantly more expanded in a non-homogeneous monolayer when they were surrounded by the molecules of lower molecular weight. In the latter case the observed conformation was close to a 2D SAW chain [30].

Rather similar results were obtained exploring another approach, which was based on SFM visualisation of brushlike molecules in a melt of polymer chains of the same nature as the side chains [31]. The melt was mixed at water subphase and then transferred onto mica for the visualisation by SFM. The dependence of the conformation of the brush-like molecules (backbones) on the length of the surrounding linear chains was demonstrated. When the surrounding molecules were rather short (oligomeric), the brush-like molecules were expanded with the corresponding scaling exponent $\nu$ of about 0.69 [31]. When the polymerisation degree of the surrounding molecules was increasing, the brush-like molecules were more and more contracted and the scaling exponent was correspondingly reducing down to the 0.48 value [31]. A set of intermediate $\nu$ values was obtained when the brush-like molecules were surrounded by the linear chains of an intermediate length.

Apart from the brush-like molecules [32], another convenient model system seemed to be nanorope chains (cross-linked worm-like micelles) [33]. Such chains had a diameter of $20 \mathrm{~nm}$ and so their morphology could be easily resolved by SFM even within dense adsorbed films. SFM visualisation with subsequent data processing detected that, in a diluted $2 \mathrm{D}$ state, the molecules were compacted in $2 \mathrm{D}$ islands with the scaling exponent value of about 0.5. This value is typical for a flat globule, i.e. a compacted conformation in a strict $2 \mathrm{D}$ case. However, in a dense $2 \mathrm{D}$ state the molecules were strongly extended and interpenetrating with the corresponding scaling exponent $\nu$ value of about 0.63 [33].

In the present paper we selected the brush-like poly(2-(2-bromopropionyloxy)ethyl methacrylate-graftpoly $(n$-butyl acrylate $)$ (p(BPEM)- $g$-p(BA)) molecules as the model system. Indeed, such molecules were easy to be visualised by SFM, and their morphology could also easily be traced by SFM even within dense adsorbed $2 \mathrm{D}$ films. We checked the macromolecular conformation (conformation of backbones) in dense LB monolayers as transferred on mica and after externally induced morphological transitions. In order to induce the transitions, we applied the previously developed method of exploring vapours of different nature as external stimuli $[34,35]$. In our previous paper [19] we performed statistical conformational analysis of the same molecules before and after vapour-induced conformational transitions at much lower surface densities. The molecules were initially deposited on mica by spin-coating as isolated objects (in a diluted state). It was found out that the macromolecules were rather extended as deposited with the scaling exponents $\nu$ of about $\sim 0.77$. But after collapse and re-extension cycle the molecules remained more compacted with the $\nu$ value of only about $\sim 0.53$ [19]. One of the reasons for the lower extension degree was related to the possible constraining effect induced by line tension (a 1D analogue of the surface tension). The line tension effects should vanish with increased overlapping of individual macromolecular islands. That may be achieved with increasing surface density of the macromolecules. 
Therefore, to check the hypothesis about line tension, it is natural to compare the macromolecular behaviour in isolated and denser states. Thus the primary goal of the present study is to reveal features of macromolecular behaviour in dense films undergoing vapour-induced conformational transitions and to relate quantitatively the directly observed conformations of single molecules in dense films to the previously investigated system with lower surface density [19].

\section{Experimental}

\subsection{Materials}

The $\mathrm{p}(\mathrm{BPEM})-g-\mathrm{p}(\mathrm{BA})$ brush molecules were prepared by ATRP-grafting $n$-butyl acrylate from a poly[2-(2-bromobutanoate)ethyl methacrylate] macroinitiator as described previously $[36,37]$. The number average polymerization degrees $\left(\mathrm{DP}_{n}\right)$ of the backbone and the side chains were $\sim 3700$ and 30 units, respectively.

The water used in the experiments was prepared with a Milli-Q Plus 185 purification system (Millipore, Germany). Chloroform of "SupraSolv" grade (Merck, Germany) was used for preparing the polymer solutions. Ethanol of analytical grade (Merck, Germany) was used as received for generating the vapour. Muscovite mica (Plano $\mathrm{GmbH}$, Germany) was applied as a substrate.

\subsection{Methods}

The macromolecules were transferred onto the substrate using the Langmuir-Blodgett (LB) technique. The horizontal deposition was performed on a Lauda FW2 Langmuir trough made of PTFE with a full surface area of $927 \mathrm{~cm}^{2}$ operated at $22^{\circ} \mathrm{C}$ using the Milli-Q water as a sub-phase. A few pieces of mica freshly cleaved from both sides were preliminary put under the water subphase on a PTFE-support. A defined volume $(0.15 \mathrm{ml})$ of the polymer solution in chloroform $(c=0.25 \mathrm{mg} / \mathrm{ml})$ was deposited at the air/water interface with fully opened barrier. Compression of the monolayers started after one hour delay to ensure homogenous spreading and possible relaxation of the polymer chains. During the compression, the surface area was reduced by $5 \%$ per minute until the surface pressure of $2 \mathrm{mN} / \mathrm{m}$ was achieved. The transfer onto the mica substrate followed by lowering the subphase level with the rate of $0.5 \mathrm{~mm} / \mathrm{min}$. The surface pressure was maintained constant at the $2 \mathrm{mN} / \mathrm{m}$ value during the transfer. After preparation and before the study, the transferred monolayers on mica were kept in laboratory room conditions (temperature of $22^{\circ} \mathrm{C}$, relative humidity of $50-60 \%$ ) for the periods from several hours and up to five days. No changes of the macromolecular morphology were observed as a result of the longer storage.

SFM images were recorded at ambient temperature by means of a "PicoSPM" device (Molecular Imaging, USA) in the acoustic mode. To introduce the vapour of ethanol or water to the atmosphere around the sample, $0.3-0.7 \mathrm{~mL}$ of the corresponding liquid were injected into the microscope environmental chamber (total volume $\sim 1.2 \mathrm{~L}$ ) through an inlet in the device without interruption of the scanning procedure. So as to replace the vapour by a dry atmosphere, nitrogen (99.999\% purity, MTI IndustrieGase AG, Germany) was abundantly blown through the chamber. Topographic SFM images were collected with an information density of $512 \times 512$ points at $1 \mathrm{~Hz}$ scanning frequency. SFM image editing and analysis were conducted by means of FemtoScan software (Advanced Technologies Center, Russia). Numerical data analysis was performed using OriginPro 7.5 software (OriginLab, USA) and homewritten analytic software.

\subsection{Data analysis}

To determine the scaling exponent $\nu$ we applied the same procedure, which was described in details in references $[17$, 19]. The end-to-end distances and contour lengths of individual macromolecules were determined from the recorded SFM micrographs with the typical size of $2.5 \times 2.5 \mu \mathrm{m}$ using FemtoScan software. The measurements were performed in a semimanual regime of the program in order to avoid any possible inaccurate identifications of individual macromolecular contours in such dense films. That means that an operator had to find one end of the particular macromolecule and then trace its contour until the next end. The program determined the thus-traced path and the direct distance between both ends. The pairs of these two values were accumulated as a statistical array of data. The number of analysed molecules $(N)$ was more than 750 for each of the two types of samples (of the macromolecules in the LB film before and after the collapse/re-spreading cycle). The resulting array of $N$ pairs of corresponding $R_{i}$ and $L_{i}$ values was sorted in an ascending order with respect to the $L_{i}$, and then an averaging procedure was performed on the $n$ successive pairs of values to yield $N / n$ values of the mean square end-to-end distance $R^{2}$ :

$$
\widehat{R}^{2}{ }_{k}=\frac{1}{n} \sum_{i=n(k-1)+1}^{n k} R_{i}^{2},
$$

the mean contour length $L$ :

$$
\widehat{L}_{k}=\frac{1}{n} \sum_{i=n(k-1)+1}^{n k} L_{i}
$$

and the corresponding standard errors of the mean values:

$$
\begin{aligned}
\Delta \widehat{R}^{2} & =\sqrt{\frac{1}{n(n-1)} \sum_{i=n(k-1)+1}^{n k}\left(\widehat{R}_{k}{ }^{2}-R_{i}^{2}\right)^{2}}, \\
\Delta \widehat{L}_{k} & =\sqrt{\frac{1}{n(n-1)} \sum_{i=n(k-1)+1}^{n k}\left(\widehat{L}_{k}-L_{i}\right)^{2}},
\end{aligned}
$$

where $k=1,2, \ldots, N / n$. In order to check the convergence of the calculated values, we varied the averaging number $n$ 
in the range of 1-90. In order to have always the integer $N / n$ ratio for different $n$ numbers, some required amount of pairs of the $R_{i}$ and $L_{i}$ values was randomly removed from the array of the $N$ pairs.

The scaling exponent $\nu$ to be determined describes the correlation between the mean-square end-to-end distance $R^{2}$ and the contour length $L$ :

$$
\widehat{R^{2}}=\text { const } \times L^{2 \nu}
$$

Thus, if the values of averaged-square end-to-end distances, $\widehat{R}^{2} k$, are plotted as a function of the corresponding mean contour lengths, $\widehat{L}_{k}$, in a double logarithmic scale, the plotted data points should be scattered around a straight line. The slope of the straight line determines the scaling exponent $\nu$ value. We applied three different fitting procedures to find the slope. The procedures differ from each other with respect to whether or not they take into account the uncertainties of the $\widehat{R^{2}}{ }_{k}$ and $\widehat{L}_{k}$ values, as given by the corresponding standard errors values: $\Delta \widehat{R}^{2}{ }_{k}$ and $\Delta \widehat{L}_{k}$.

The first procedure (procedure I) consists of a linear regression of a set of $N / n$ data points in a double logarithmic scale and does not take into account any weight given by the standard error values (simple least-squares method). This approximation was performed using a standard option of the Origin software for a simple linear fit. The second procedure (procedure II) consists of a weighted linear regression of a set of $N / n$ data points in a double logarithmic scale with weights given by the $\Delta \widehat{R}^{2} k$ error values (weighted least-squares method). This approximation was also performed using another standard option of the Origin software for an error-weighted linear fit. The third procedure (procedure III) consists of the numerical minimization of the following weighted chi-square merit function with respect to "const" and $\nu$ values $[17,19]$ :

$$
\begin{aligned}
& \chi^{2}(\text { const }, \nu)= \\
& \sum_{i=1}^{N / n}\left(\frac{\left(\widehat{R}^{2}{ }_{i}-\text { const } \times \widehat{L}_{i}^{2 \nu}\right)^{2}}{\left(\operatorname{const} \times 2 \nu \widehat{L}_{i}^{2 \nu-1} \Delta \widehat{L}_{i}\right)^{2}+\left(\Delta \widehat{R}^{2}{ }_{i}\right)^{2}}\right) .
\end{aligned}
$$

Here, both the uncertainties of the averaged $\widehat{R}^{2} k$ and $\widehat{L}_{k}$ values are properly taken into account. The uncertainties are given by the corresponding standard errors: $\Delta \widehat{R}^{2}{ }_{k}$ and $\Delta \widehat{L}_{k}$ of the mean values, see equations $(3,4)$. In order to perform such an analysis following procedure III, we wrote a simple calculating software. As far as only procedure III properly accounts for both types of uncertainties of the $\widehat{R}_{k}^{2}$ and $\widehat{L}_{k}$ values, we consider this procedure as the most correct. This question was addressed before in reference [19]. In the analysis, we take into account the $\nu$ values, determined according to procedure III. The results for procedure I and procedure II are also given for comparison. They do not differ significantly.

\section{Results}

\subsection{SFM visualisation of macromolecules in dense LB film, vapour-induced morphology changes}

The general macromolecular contours of the $\mathrm{p}(\mathrm{BPEM})-g$ $\mathrm{p}(\mathrm{BA})$ brush molecules (backbones) can be clearly seen in the dense LB film transferred onto mica (Fig. 1a). Inside the film, the macromolecular backbones are separated from each other by a fixed distance of about $35 \mathrm{~nm}$. This separation is apparently determined by the sizes of coronas of the side chains surrounding the backbones, as discussed in reference [34]. The heights of the main macromolecular profiles (backbones) in the film above the surrounding areas (occupied by the side chains) are in range of $0.5-0.9 \mathrm{~nm}$. The intersections of the macromolecules are strongly reduced (Fig. 1a), most macromolecular contours are separated from their neighbours. When one traces a particular macromolecular contour in the observed labyrinth-like structure of Figure 1a, one most probably finishes in some cul-de-sac. In this sense the degree of percolation of the macromolecular paths (backbones) is rather small. For dense two-dimensional films, the question about the degree of overlapping of different chains is always interesting [1]: whether different macromolecular contours are strongly interpenetrating and overlapping or, on the contrary, are segregated and overlap only slightly. For our specially prepared system, one can observe no segregation of the macromolecular contours. On the contrary, the macromolecules are strongly interpenetrating. Even before any measurements of the scaling exponent $\nu$, one may already clearly notice that the degree of macromolecular extension is rather high. In some places of the film the macromolecules are even perfectly straight. Does this mean that the chains are here in a persistent regime and should demonstrate $\nu$ values close to 1 ? This is hardly the case, since a closer look shows that the persistence here is only apparent. Indeed there are straight sections of the macromolecules but they are quite often followed by abrupt kinks not compatible with real persistence.

Previously, we reported that by means of an exposure of the $\mathrm{p}(\mathrm{BPEM})-g-\mathrm{p}(\mathrm{BA})$ molecules adsorbed on mica to different vapours it was possible to induce either collapse or re-expansion of the single macromolecules, depending on the nature of the vapour $[34,35]$. The vapourinduced conformational transitions were observed in real time by SFM. It was established that vapours of strongly amphiphilic compounds (like alcohols) induced collapse, whereas vapours of polar compounds (like water) induced re-expansion of the previously collapsed macromolecules. It was explained by competition in spreading between somewhat amphiphilic polymer chains and co-adsorbed low molecular weight compounds [35]. If the polymer was more amphiphilic than the surrounding co-adsorbed layer (like water) then the polymer spreads maximising an occupied surface area per macromolecule. If the polymer was less amphiphilic (as compared, e.g., to ethanol), it was removed from the interface and forced to compact into 3D globules, minimising an occupied surface area 

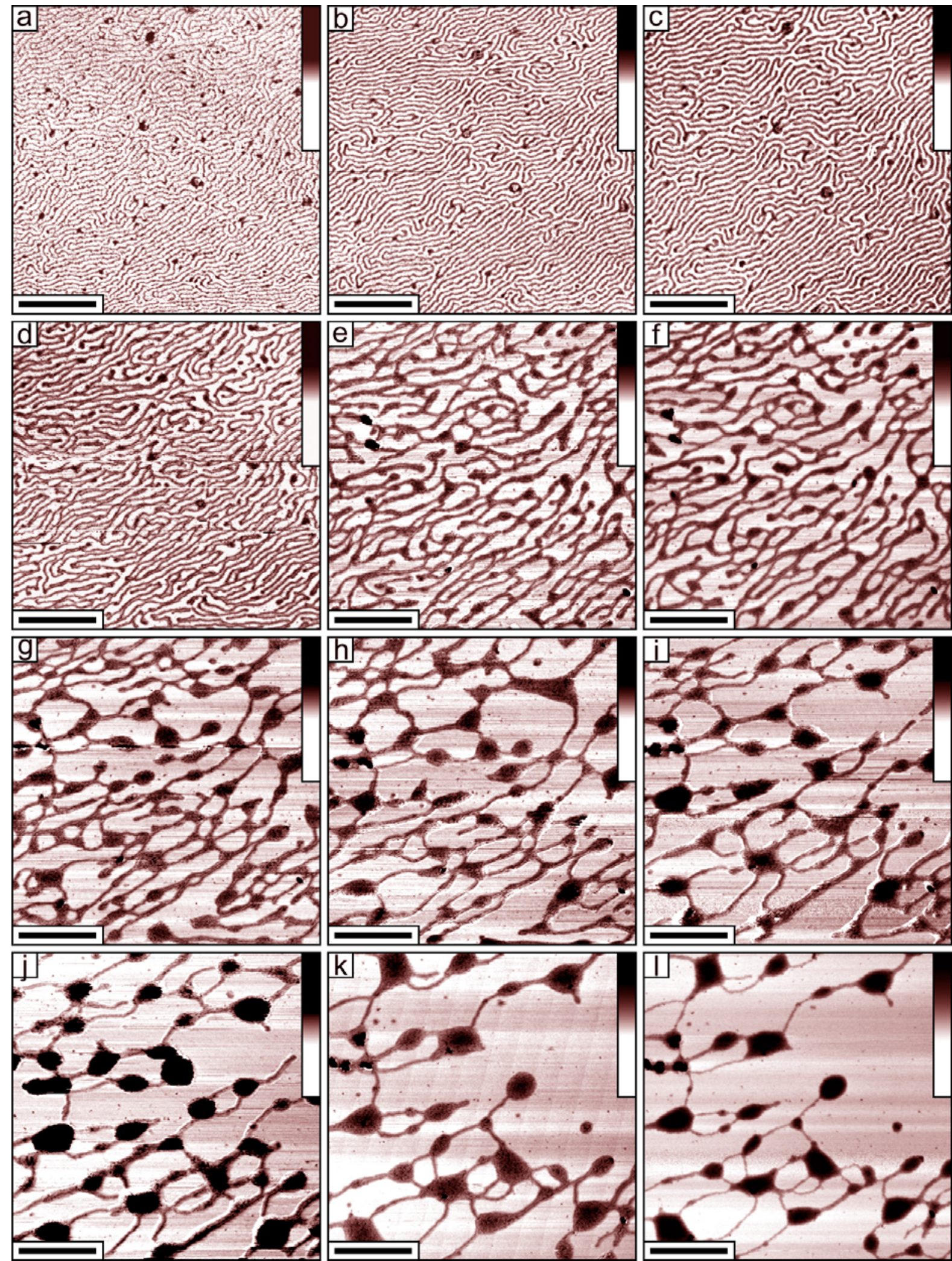

Fig. 1. Collapse of LB film of brush-like p(BPEM)- $g$-p(BA) molecules induced by exposure to ethanol vapour: SFM visualization in real-time regime. (a) Initial image of the LB film as deposited, single molecules in the dense film can be observed; (b-k) the

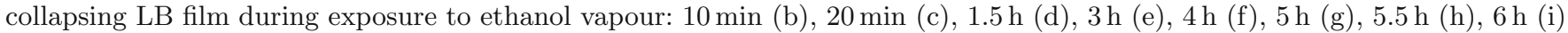
$6.5 \mathrm{~h}(\mathrm{j}), 7.5 \mathrm{~h}(\mathrm{k})$ after injection of ethanol into the environmental chamber; (l) final morphology frozen by purging the chamber with nitrogen. Scan size: $2 \times 2 \mu \mathrm{m}^{2}$, bar size: $500 \mathrm{~nm}$, height scale: $10 \mathrm{~nm}$. 
per macromolecule. Later on it was shown that this effect was rather a general one and could be observed for different amphiphilic polymer molecules (including linear P2VP chains [38]) on different substrates (oxidised silicon, strontium titanate [39]).

Thus we have subjected the dense LB film of the $\mathrm{p}(\mathrm{BPEM})-g$-p(BA) molecules subsequently to vapours of ethanol and water in order to check the matter of vapourinduced morphology changes to be observed in real time with SFM. The exposure to ethanol vapour has induced a collapse of the LB film (Fig. 1b-k). The behaviour of the macromolecules in the collapsing film is rather peculiar. Initially, the height of the macromolecular profiles above the surrounding has started to increase up to $1.3-1.7 \mathrm{~nm}$ (Fig. 1b) after $10 \mathrm{~min}$ of the exposure to ethanol vapour and further up to $1.6-1.9 \mathrm{~nm}$ (Fig. 1c) after $20 \mathrm{~min}$ of the exposure. This is similar to the behaviour of single individual collapsing macromolecules, as reported before [34]. But after that the neighbouring parallel segments of different macromolecules have started to joint together, see Figure 1d, which has been obtained after $1.5 \mathrm{~h}$ of the exposure to ethanol vapour. This is different from what to be observed for single individual collapsing macromolecules, where formation of centres of compaction at the ends of single molecules has been reported [34]. In the dense film the collapse is rather an aggregation of neighbouring molecules which results in increasing the percolation degree. This aggregation proceeds further (Fig. 1e, f: $3-4 \mathrm{~h}$ of exposure) with increasing the percolation degree and results in the formation of an interconnected $2 \mathrm{D}$ polymer network. At the same time, the formation of the centres of compaction is observed in the nodes of the network (Fig. 1e, f). Such centres are consuming more and more aggregated polymer filaments (Fig. $1 \mathrm{~g}-\mathrm{k}$ : $5-7 \mathrm{~h}$ of exposure) and are increasing in size. At the final image of the collapse process (Fig. 1l, the morphology was frozen by nitrogen purging) one can observe many separated big blobs with a height of about $4-6 \mathrm{~nm}$. Comparing the initial number of the macromolecules at Figure 1a and the final number of the blobs at Figure 1l, one can conclude that each blob contains several different molecules $(5-10)$. The blobs were interconnected with remaining (not collapsed) polymer filaments. We expect that the reason for the incomplete collapse is slow kinetics. Possibly, for longer exposure times the collapse would be more complete. As far as the blobs are separated, there are significant surface areas without any polymer between them. Thus, the polymer material has been efficiently expelled from the interface by the amphiphilic ethanol molecules. Therefore, exposure of a dense polymer LB film on mica to ethanol vapour results in the collapse of the film.

After that, we have subjected the collapsed LB film of the $\mathrm{p}(\mathrm{BPEM})-g-\mathrm{p}(\mathrm{BA})$ molecules to the water vapour, in order to check whether the collapse is reversible. Indeed, we have found out that the exposure to water vapour induced re-spreading (decollapse) of the LB film (Fig. 2b-k). The behaviour of the expanding macromolecules here resembles the previously reported one for single individual molecules. The blobs are untwining and the single molecules are crawling out of them spreading over the surface. One can notice that the macromolecules as crawling out of the globules are highly curved (Fig. 2d-i). Finally, after $12 \mathrm{~h}$ of exposure to water vapour, the motion of the macromolecules apparently ceases (Fig. 2k). One can observe that finally there are some remaining blobs, which have not untwined completely (Fig. 2k). At the same time some areas of mica remain to be unoccupied. Incomplete spreading may be indicative of some macromolecular friction on the mica substrate. Thus the reversibility is only partial. Besides, in the final state the macromolecules remain to be more curved (Fig. 2l) as compared to the initial state as deposited (Fig. 1a). Here also the macromolecular contours are mostly interpenetrating and rather extended as far as only some of them indicate partial segregation.

\subsection{Determination of scaling exponent values}

We have performed a numerical analysis of the visualised macromolecular contours (backbones) in order to determine the scaling exponent $\nu$ for single $\mathrm{p}(\mathrm{BPEM})-g-\mathrm{p}(\mathrm{BA})$ molecules inside the dense LB film as deposited and after a collapse/re-extension cycle. For that we have followed the previously developed method of the analysis as described in the Experimental section. Rather large numbers of different macromolecular profiles have been analysed $(N>750)$ for both types of samples (before and after the collapse). The mean-square end-to-end distances obtained for an averaging depth $n=40$ are plotted as a function of the corresponding mean contour lengths in double-logarithmic scale in Figure 3. Here two sets of the data points are presented: for the molecules in the film as deposited (A), and for the same molecules after a cycle of collapse/re-spreading of the film (B). One can see that both data sets can be well approximated by linear fits: corresponding straight lines are indicated, as obtained using procedure III (see the Experimental section). One can see that the points of the first data set (A, as deposited) are located above the points of the second one (B, after the cycle). This is an indication of a reduced, in general, end-to-end distances as a result of the cycle of the morphology transformation of the film. And indeed, the measured mean end-to-end distance (defined as a square root of the mean-square end-to-end distance $\sqrt{\sum R_{i}^{2} / N}$ ) has been reduced by $23 \%$ from the initial $350 \mathrm{~nm}$ value (as deposited) down to the $270 \mathrm{~nm}$ value (after the cycle). The number average contour length $\left(L_{n}=\sum L_{i} / N\right)$ has been also slightly reduced by $10 \%$ from the initial $540 \mathrm{~nm}$ value (as deposited) down to the $490 \mathrm{~nm}$ values (after the cycle). The reduction of the contour lengths may result either from contraction of the backbones or from some scission [40] of them during the collapse/re-spreading of the film.

The significantly stronger reduction of the mean endto-end distance is indicative of a higher compactness degree of the macromolecules after the cycle. In equation (5) the $\nu$ value is an example of global parameters describing the type of statistics. The prefactor "const" is a local parameter depending on the properties of the particular chains such as stiffness, interaction with substrate, etc. [1]. 

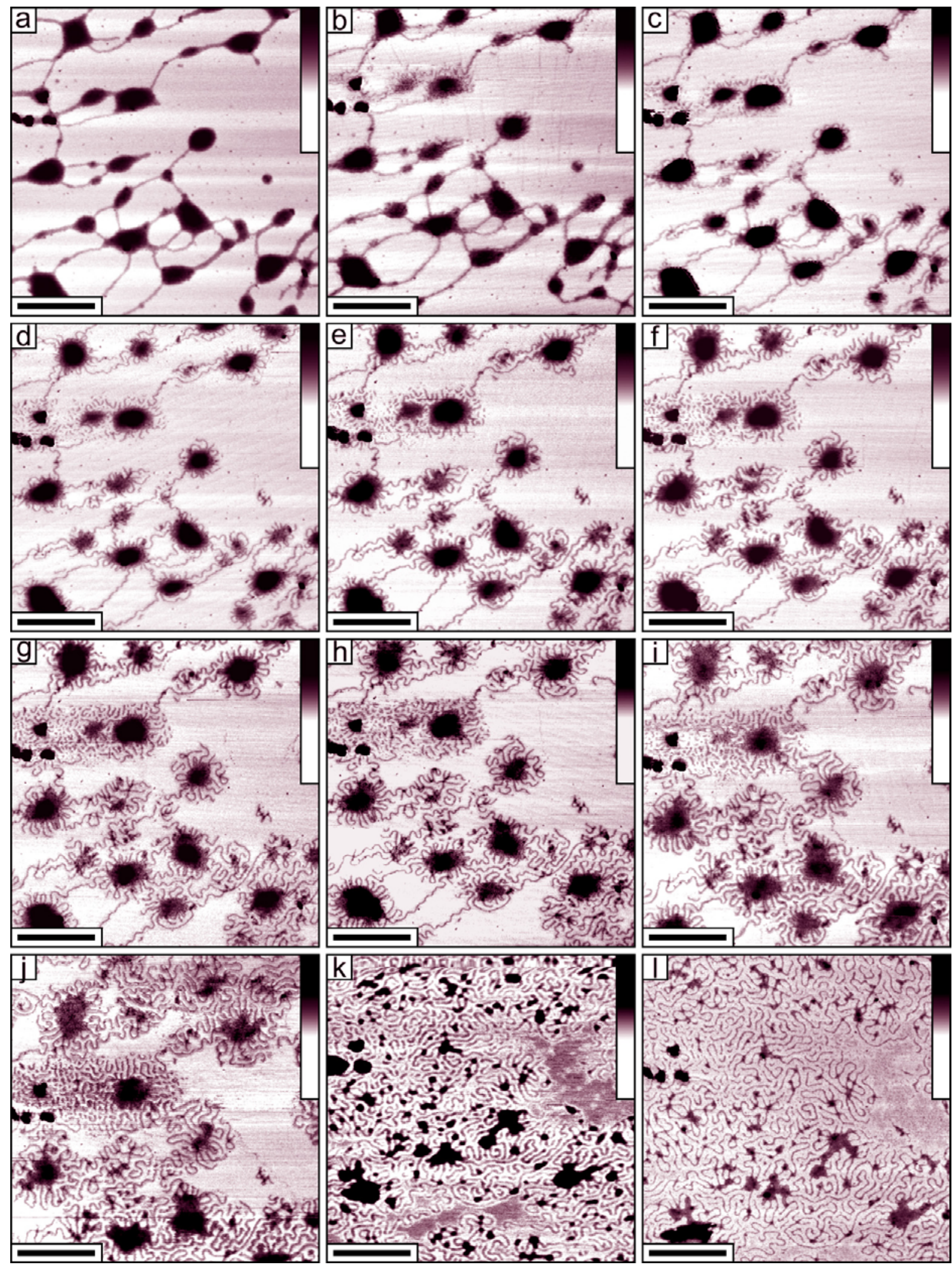

Fig. 2. Re-spreading of the collapsed film of brush-like $p(B P E M)-g-p(B A)$ molecules induced by exposure to water vapour: SFM visualization in real-time regime. (a) Starting image of the pre-collapsed LB film (Fig. 1); (b-k) spreading of the film during exposure to water vapour: $10 \mathrm{~min}$ (b), $20 \mathrm{~min}$ (c), $30 \mathrm{~min}$ (d), $40 \mathrm{~min}$ (e), $50 \mathrm{~min}$ (f), $60 \mathrm{~min}$ (g), $70 \mathrm{~min}$ (h), $1.5 \mathrm{~h} \mathrm{(i),}$ $2 \mathrm{~h}(\mathrm{j}), 12 \mathrm{~h}(\mathrm{k})$ after injection of water into the environmental chamber; (l) final morphology after purging the chamber with nitrogen, single molecules in the dense film can be observed. Scan size: $2 \times 2 \mu \mathrm{m}^{2}$, bar size: $500 \mathrm{~nm}$, height scale: $10 \mathrm{~nm}$. 


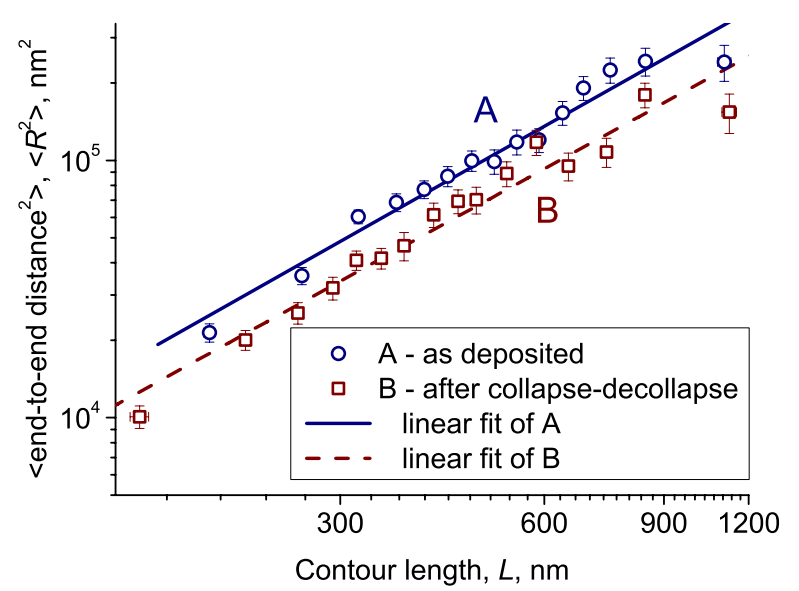

Fig. 3. Correlation between mean-square end-to-end distances ${\widehat{R^{2}}}_{k}$ and contour lengths $\widehat{L}_{k}$, for p(BPEM)- $g$-p (BA) brush-like molecules in the dense LB film: (A) before ethanol-vapourinduced collapse of the film, (B) after the water-vapourinduced re-spreading of the film. Experimental points: each data point is obtained as a result of an averaging procedure applied according to equations (1) and (2) for $n=40$. Error bars are determined as standard errors of the mean values according to equations (3) and (4). An approximating straight line was obtained using procedure III, see the Experimental section.

The reduction of the prefactor "const" as a result of the collapse-decollapse transformation may be explained by a more equilibrated redistribution of the side chains determining the local macromolecular flexibility [41]. From Figure 3 one can see that the slope of the approximating linear fits has not changed after the collapse/decollapse cycle. That means that the scaling exponent $\nu$ has not changed. Does this mean the same type of macromolecular statistics at the initial and the final stage? This question is to be addressed in the Discussion section taking into account the obtained numbers.

The values of the $\nu$ exponent obtained using all three fitting procedures (procedure I, II, and III, see the Experimental section) are presented in Table 1 for different averaging numbers $n$ (1-90). The choice of the averaging number $n$ seems to be important with respect to the convergence of the resulting calculated values. Reduced scattering of the data points and suppressed fluctuations of the determined $\nu$ values are obtained only when $n>20$. The mean values reported in the last row of the table have been obtained by an averaging of the $\nu$ values in the corresponding columns for the whole range of the $n$ numbers from 20 to 90 . The calculated standard deviations of all the mean values in Table 1 are about 0.01 and are smaller than the typical 0.03 values describing the errors of approximations (the errors determined by scattering of the data points being approximated). Thus the uncertainties of the $\nu$ in Table 1 should be determined by these 0.03 values. One can see that there are some differences for the $\nu$ values obtained using three different fitting procedures (procedure I, II, and III). We consider procedure III to be
Table 1. Values of the scaling exponent $\nu$ determined using different averaging numbers $n$ and different fitting procedures (procedure I, II, and III, see the Experimental section). The mean values in the last row are obtained by averaging the $\nu$ values for $n>20$.

\begin{tabular}{l|ccc|ccc}
\hline \multirow{2}{*}{} & \multicolumn{3}{|c|}{$\mathrm{p}$ (BPEM)-g-p(BA), } & \multicolumn{3}{c}{$\mathrm{p}(\mathrm{BPEM})-g$-p(BA), } \\
& \multicolumn{3}{|c}{$N=760$} & \multicolumn{2}{c}{ re-expanded in water } \\
& \multicolumn{3}{|c}{ vapour, $N=790$} \\
$n$ & Pr. I & Pr. II & Pr. III & Pr. I & Pr. II & Pr. III \\
\hline 1 & 0.55 & 0.55 & 0.50 & 0.67 & 0.67 & 0.59 \\
3 & 0.66 & 0.39 & 0.78 & 0.57 & 0.78 & 0.66 \\
5 & 0.70 & 0.81 & 0.71 & 0.72 & 0.68 & 0.66 \\
10 & 0.71 & 0.76 & 0.69 & 0.73 & 0.72 & 0.69 \\
15 & 0.71 & 0.77 & 0.69 & 0.73 & 0.75 & 0.71 \\
20 & 0.72 & 0.79 & 0.75 & 0.73 & 0.76 & 0.73 \\
25 & 0.72 & 0.78 & 0.74 & 0.72 & 0.76 & 0.71 \\
30 & 0.73 & 0.78 & 0.75 & 0.72 & 0.75 & 0.73 \\
40 & 0.74 & 0.79 & 0.76 & 0.72 & 0.75 & 0.73 \\
50 & 0.74 & 0.78 & 0.76 & 0.73 & 0.78 & 0.74 \\
60 & 0.74 & 0.78 & 0.76 & 0.73 & 0.76 & 0.73 \\
75 & 0.73 & 0.76 & 0.74 & 0.74 & 0.78 & 0.76 \\
90 & 0.73 & 0.75 & 0.74 & 0.72 & 0.73 & 0.72 \\
\hline mean & 0.73 & 0.78 & 0.75 & 0.73 & 0.76 & 0.73 \\
\hline
\end{tabular}

the most valid one. Indeed, only this procedure ensures that both types of uncertainties of the averaged $\widehat{R^{2}} k$ and $\widehat{L}_{k}$ values are properly taken into account for the fitting. These uncertainties are given by the corresponding standard errors: $\Delta \widehat{R}^{2}{ }_{k}$ and $\Delta \widehat{L}_{k}$ of the mean values. Therefore we accept that the scaling exponent $\nu$ value is equal to 0.75 for the macromolecules in the initial dense LB film (as deposited) and is equal to 0.73 for the same macromolecules after a cycle of the vapour-induced collapse and subsequent re-spreading of the film. Only one slope is observed for the whole range of the contour lengths and in spite of the apparent persistence of some chains in Figure 1a the slope is well below 1. Clearly, upon averaging the apparent persistence of some chains is cancelled by the sharp turns of the others.

\section{Discussion}

Both determined values of the $\nu$ exponent are rather large and close to the $0.75(3 / 4)$ value of the 2D SAW statistics. Our $\nu$ values are the largest among all the published in the literature for macromolecules in dense 2D monolayers as obtained experimentally, theoretically or using computer simulations, see the Introduction. Regarding the obtained 0.75 initial $\nu$-value for the macromolecules in the dense LB film as deposited, we do not see here any possibility for an irrelevance. Indeed, it was shown before that the same macromolecules as deposited from chloroform solutions on mica [19] or silicon [39] substrates at low surface density 
adopted a similar conformation with the same scaling exponent of about 0.75 . The set of data points A on Figure 3 coincides perfectly well with the set of data points on Figure 4a of the reference [19] obtained for the same molecules as deposited on mica in a more diluted state (as isolated chains). That means that not only the slope $\nu$ but even the prefactor "const" of equation (5) is the same for both cases of the deposited molecules with significantly different surface concentration. The reason for the invariance of the macromolecular conformation on the surface density is unclear. Besides, the 2D SAW statistics is not applied to the macromolecules in dense films. Indeed, in this model there is general assumption that every step of a random walker going along the $2 \mathrm{D}$ self-avoiding path is randomly oriented [9]. This assumption cannot be valid in a dense state with strongly interpenetrating paths. We explain this singularity by the hypothesis that the macromolecules in the dense film may "remember" their parent conformation just as adsorbed on water subphase before compression.

Indeed, one can suppose that as deposited from chloroform solution at the water subphase the macromolecules adopt the same 2D SAW conformation as being deposited on mica (with the same $0.75 \nu$ value). Taking into account the amount of the polymer deposited on the water subphase and the surface area of LB trough, one can calculate that the surface density of the macromolecules with a mean contour length of $540 \mathrm{~nm}$ on the water subphase is ca. 30 at $(\mu \mathrm{m})^{2}$. This is about 3 times larger than in typical experiments with isolated molecules deposited on mica [19], but still comparable.

As the compression starts the macromolecules begin to contact each other and are forced to pack more and more tightly. But their contour lengths are obviously unaffected by the compression. Only end-to-end distances are distorted due to some deformations of the initial macromolecular islands at the subphase. If the system is able to re-equilibrate during the slow compression, the macromolecules may reorganise and indicate different values of the end-to-end distances at the end of compression. But we believe that in the current system the equilibration during the compression step is strongly suppressed. Indeed, the redistribution of the side chains relatively to the main chain is required for the equilibration [41]. Such redistribution implies many overflips of the adsorbed side chains. The probability of the overflip event is supposed to be rather small.

As a result of the deformations without re-equilibration, the end-to-end distances may either slightly increase or decrease, independently of the macromolecular contour lengths. After averaging, such changes are mostly mutually compensated. Accordingly, after the compression and transfer of the film onto a substrate, the scaling exponent remains still to be close to the same 0.75 value, as we have experimentally determined.

The macromolecular profiles are strongly interpenetrating and highly extended in the transferred film (Fig. 1a). This conformation differs significantly from typical conformations with lower $\nu$-values, particularly from a conformation with a scaling exponent of 0.5 . In a strict
$2 \mathrm{D}$ case the scaling exponent of 0.5 corresponds either to a compacted $2 \mathrm{D}$ flat globule, or to some other conformations depicted in reference [19] with fixed packing density of monomer units (under the assumption that each monomer unit occupies the same surface area, the macromolecular size scales as $\widehat{R^{2}} \sim S \sim L$, as far as the surface area $S$ scales as $L$ and hence $\nu \sim 1 / 2$ ). Such conformations are rather peculiar and differ from what is observed in Figure 1a. The highly extended conformation observed here should be described by rather large scaling exponent $\nu$ values, and this fact justifies the determined $0.75 \nu$ value.

Therefore the initial state of the prepared dense films (see Fig. 1a) should be considered as a special nonequilibrium "frozen" state with the macromolecules having certain memory about the parent 2D SAW conformation adopted during adsorption on water subphase before compression. The spontaneous equilibration of the compressed and transferred film is apparently significantly suppressed. It is not observed during storage of the samples for at least several days. As far as there is no equilibration for the compression step, the obtained $\nu$ value cannot be regarded as an indicator of the type of statistics of the macromolecules in the dense films. It is only an indicator of the type of statistics for the macromolecules equilibrated during the adsorption step at the water subphase in a more dilute state.

The observed restoration of the rather high scaling exponent $\nu$ value after a collapse/decollapse cycle seems to be more intriguing. Indeed, it was shown before that more spatially separated single individual macromolecules at lower surface densities re-extended to a smaller degree after the collapse: the restored scaling exponent value was found to be only about 0.53 [19]. What could be the possible reasons for a higher degree of re-extension of exactly the same macromolecules just at increased surface density? On can propose two mechanisms of the higher extension.

The first one comprises the dependence of the ultimate $2 \mathrm{D}$ conformation on the intermediate conformation of the molecule in $3 \mathrm{D}$ at the collapse stage. Indeed, in the case of the re-extension of the single macromolecule from the single $3 \mathrm{D}$ globule [19], the compact 2D conformation with $\nu=0.53$ comes from the compact conformation in the $3 \mathrm{D}$ globule whose size $R_{3 \mathrm{D}}$ scales with the contour length $L$ as $R_{3 \mathrm{D}} \sim L^{1 / 3}$. In such $3 \mathrm{D}$ conformation, the molecule is strongly curved and the curved parts of the brush are characterized by asymmetric distribution of the side chains relative to the backbone, i.e., convex regions have higher fraction of the side chains than the concave ones. It is supposed that during adsorption of the brush the local asymmetry of the side chains is retained and the $2 \mathrm{D}$ brush also adopts strongly curved conformation. One more argument in favour of the asymmetric distribution of the side chains of the 2D brush is dictated by thermodynamics: asymmetrically distributed side chains have smaller free energy of stretching than the symmetrically distributed ones. This effect is known as spontaneous curvature of $2 \mathrm{D}$ brushes $[42,41,43]$. Line tension of the single $2 \mathrm{D}$ brush also promotes compaction. 
In the case of LB films transferred on mica, the collapse of the molecules results in the formation of aggregates interconnected by polymer filaments, see Figure 11. Each aggregate contains few macromolecules, i.e., the aggregates are the globules whose aggregation number is larger than unity. It is obvious that if the size of the aggregate $R_{3 \mathrm{D}}$ exceeds the Gaussian size of the brush, $\sim L^{1 / 2}$, the macromolecules in the aggregate have a Gaussian statistics like in a 3D melt. If the size of the aggregate is smaller than the Gaussian one, the exponent $\nu$ belongs to the range $1 / 3<\nu<1 / 2$. Thus, we can conclude that in the case of the dense LB film, the collapsed (3D) macromolecules in multimolecular globules have more elongated conformations than single collapsed ones. Due to such elongation, each molecule in the multimolecular aggregate is less curved than the molecule in unimolecular globule and the degree of the asymmetry of the side chains is less pronounced. It means that after re-adsorption the $2 \mathrm{D}$ conformation of the molecules in the film has to be more extended because of a more symmetric distribution of the side chains and shielding of the line tension.

Despite the simplicity, the above-proposed reasoning can not quantify the end-to-end distance after readsorption. To do so, let us propose a second mechanism of extension of the 2D macromolecules, which does not take into account the specific chemical structure of the comb-like macromolecules and should be valid for any linear flexible chains forming monomer-thick films and collapsing in certain vapours. As we can see in Figure 1, the collapse of the brushes in the LB film proceeds through the formation of mesoscopic droplets (aggregates) interconnected by polymer filaments rather than the formation of one big (macroscopic) droplet which thermodynamically is more favourable because it would possesses a lowest surface energy. Therefore, the existence of the finite-size aggregates is a metastable state, but this state can exist for an infinitely long time (at least for a few days) because the distance between the aggregates is large enough, they are not so mobile due to the friction with the substrate, and the thermodynamic fluctuations are rather weak to promote coalescence of the aggregates. Thus, we may assume that the aggregates are in quasiequilibrium. The natural question is: what does control the size and the aggregation number of the aggregates? Keeping in mind that there is only one mesoscopic length scale in the LB film (the end-to-end distance $R_{2 \mathrm{D}}$ of the molecule), one can assume that only those macromolecules which belong to the circle of the diameter $R_{2 \mathrm{D}}$ form the aggregate after being collapsed. It means that the higher $2 \mathrm{D}$ stretching of the molecules in the film, the larger the aggregates. In other words, there is a competition between elasticity of the chains in 2D (the stretching is unfavourable) and the surface energy in 3D (bigger aggregates have smaller surface energy). Namely, this competition determines the optimum stretching of the chains in the film. Let us calculate this stretching assuming that the end-to-end distance scales as $R_{2 \mathrm{D}}=\lambda^{1-\nu} L^{\nu}$, where $L$ and $\lambda$ are the apparent contour length and the persistence length of the brush, respectively. Here $\nu$ is the still unknown parameter. The persistence length of $2 \mathrm{D}$ brush with fixed (quenched) dis- tribution of the side chains with respect to the backbone has a cubic dependence on the number of segments in the side chain $M, \lambda \approx a M^{3}[44,45]$, where $a$ is the linear size of the segment. Within the Flory theory, the elastic free energy of the 2D molecule takes the form

$$
\frac{F_{\mathrm{el}}}{k_{\mathrm{B}} T} \approx \frac{R_{2 \mathrm{D}}^{2}}{\lambda L} \approx\left(\frac{L}{\lambda}\right)^{2 \nu-1}
$$

where $k_{\mathrm{B}} T$ is the thermal energy. The dense-packing conditions of the molecules in the film and the aggregate

$$
a R_{2 \mathrm{D}}^{2} \approx Q D L a \approx R_{3 \mathrm{D}}^{3}
$$

determine the aggregation number $Q \approx \lambda^{2-2 \nu} L^{2 \nu-1} / D$ and the surface tension of the aggregate per molecule

$$
\frac{F_{\mathrm{sur}}}{k_{\mathrm{B}} T} \approx \frac{\gamma R_{3 \mathrm{D}}^{2}}{k_{\mathrm{B}} T Q} \approx \bar{\gamma}\left(\frac{\lambda}{a}\right)^{2 / 3}\left(\frac{L}{\lambda}\right)^{1-2 \nu / 3}
$$

Here $D$ is the width of the $2 \mathrm{D}$ brush, $D \approx a M \approx$ $a(\lambda / a)^{1 / 3}[44]$, and $\bar{\gamma}=\gamma a^{2} / k_{\mathrm{B}} T \sim 1$ is the dimensionless surface tension coefficient at polymer/vapor surface. According to our assumption that the initial 2D conformation of the molecule in the film determines the size of the $3 \mathrm{D}$ aggregate, the optimum $2 \mathrm{D}$ stretching is calculated by minimization of the sum of the free energies of the film and the aggregates (the free energy per cycle) over the exponent $\nu$. Taking into account that the interfacial energy of the film does not depend on $\nu$ and that the confinement free energy [46] of the molecules in the aggregate is much smaller than the surface energy, the minimization is applied only to the sum of equations (7) and (9):

$$
\nu=\frac{3}{4}+\frac{3}{8} \cdot \frac{\ln (\bar{\gamma} / 3)+2 / 3 \cdot \ln (\lambda / a)}{\ln (L / \lambda)} .
$$

Thus, in the limit of infinitely long molecule, $L \rightarrow \infty$, the exponent tends to the constant value $\nu \rightarrow 3 / 4$, which nicely corresponds to the measured one, $\nu \approx 0.73$. Of course, the crucial assumption of the second mechanism of the 2D elongation is that the aggregates do not coalesce. Otherwise, the realization of true equilibrium would result in the Gaussian conformations in the LB film, and in the macroscopic droplet after the molecules are collapsed.

The proposed two mechanisms of elongation of strongly adsorbed (2D) macromolecules are not necessarily alternative and can coexist with each other. The aboveobtained $\nu$ value is only formally coinciding with the $2 \mathrm{D}$ SAW exponent values. Thus, it cannot be regarded as an indicator of the type of statistics for the macromolecules in the dense films after re-extension.

\section{Conclusion}

We observed single brush-like macromolecules in dense monolayer LB films transferred on mica substrate. The initial state of the films was prepared by deposition of the macromolecules on water subphase with subsequent 
lateral compression and horizontal transfer onto mica. In such films the macromolecules demonstrated a high degree of extension. Their conformation is described by the scaling exponent $\nu$ equal to 0.75 . This value is normally indicative of 2D SAW statistics corresponding to isolated chains. We related the observed value to the suppressed equilibration of the macromolecules during the compression step. In this sense the macromolecules in the films kept some memory about parent conformation as deposited on water subphase. In such more diluted state the 2D SAW exponent might be expected, provided the macromolecules equilibrate during the adsorption.

In the compressed film the macromolecular intersections are suppressed and the degree of percolation is very small: individual macromolecular trajectories mostly finish in cul-de-sacs. The exposure of the LB film to ethanol vapour induces its collapse transformation: initially into well-percolated 2D network and finally into segregated adsorbed aggregates still interconnected by some remaining polymer filaments. Subsequent exposure of the collapsed film to water vapour induces its re-spreading. But the restoration of the initial film morphology is not fully complete: at the final stage of the re-spreading process there are still some partially compacted aggregates remaining.

Individual macromolecules in the re-spread film are also re-extended. In general, they are more compacted as compared to the initial state (the mean end-to-end distance is smaller). The corresponding scaling exponent $\nu$ is equal to 0.73 , which is slightly smaller as compared to the initial one. Yet, it is larger then the value which has been observed previously for the same macromolecules as re-extended at smaller surface density on mica. Two possible explanations of the higher extension of the macromolecules at higher surface densities are given: one of them takes into account a "memory" about the intermediate conformation in the collapsed state (3D aggregates), and another one takes into account a balance between the surface energy of the 3D aggregates and the stretching free energy of the molecules in the film. We proposed a theoretical model explaining that the observed $\nu$ value for the re-extended molecules in the dense film only formally coincides with the 2D SAW exponent.

M. Möller would like to thank the Körber-Stiftung for its generous support. M.O. Gallyamov acknowledges the support from the Russian Foundation for Basic Research, Project No. 07-0300996-a. Financial support by the Deutsche Forschungsgemeinschaft within the SFB 569 (A.R. Khokhlov and I.I. Potemkin), Russian Academy of Sciences within the programme of the Division of Chemistry (I.I. Potemkin and M.O. Gallyamov), and the Dynasty Foundation (I.I. Potemkin) is gratefully acknowledged.

\section{References}

1. P.-G. de Gennes, Scaling Concepts in Polymer Physics (Cornell University Press, Ithaca, 1970).

2. R. Vilanove, F. Rondelez, Phys. Rev. Lett. 45, 1502 (1980).
3. F. Monroy, H.M. Hilles, F. Ortega, R.G. Rubio, Phys. Rev. Lett. 91, 268302 (2003).

4. R. Vilanove, D. Poupinet, F. Rondelez, Macromolecules 21, 2880 (1988).

5. A. Takahashi, A. Yoshida, M. Kawaguchi, Macromolecules 15, 1196 (1982).

6. A. Takahashi, A. Yoshida, M. Kawaguchi, Macromolecules 16, 956 (1983).

7. H.M. Hilles, F. Ortega, R.G. Rubio, F. Monroy, Phys. Rev. Lett. 92, 255503 (2004).

8. G.T. Gavranovic, J.M. Deutsch, G.G. Fuller, Macromolecules 38, 6672 (2005).

9. D.S. McKenzie, Phys. Lett. C: Phys. Rep. 27, 36 (1976).

10. M. Joanicot, B. Revet, Biopolymers 26, 315 (1987).

11. B.T. Stokke, D.A. Brant, Biopolymers 30, 1161 (1990).

12. B. Maier, J.O. Rädler, Phys. Rev. Lett. 82, 1911 (1999).

13. B. Maier, J.O. Rädler, Macromolecules 33, 7185 (2000).

14. P.-K. Lin, C.-C. Fu, Y.-L. Chen, Y.-R. Chen, P.-K. Wei, C.H. Kuan, W.S. Fann, Phys. Rev. E 76, 011806 (2007).

15. F. Valle, M. Favre, P.D.L. Rios, A. Rosa, G. Dietler, Phys. Rev. Lett. 95, 158105 (2005).

16. E. Ercolini, F. Valle, J. Adamcik, G. Witz, R. Metzler, P.D.L. Rios, J. Roca, G. Dietler, Phys. Rev. Lett. 98, 058102 (2007).

17. M.O. Gallyamov, S.G. Starodubtsev, T.P. Bragina, L.V. Dubrovina, I.I. Potemkin, O. Marti, A.R. Khokhlov, Macromol. Chem. Phys. 208, 164 (2007).

18. Y. Roiter, S. Minko, J. Phys. Chem. B 111, 8597 (2007).

19. M.O. Gallyamov, B. Tartsch, P. Mela, I.I. Potemkin, S.S. Sheiko, H. Börner, K. Matyjaszewski, A.R. Khokhlov, M. Möller, J. Polym. Sci. B: Polym. Phys. 45, 2368 (2007).

20. F. Kühner, M. Erdmann, H.E. Gaub, Phys. Rev. Lett. 97, 218301 (2006).

21. B. Duplantier, J. Phys. A: Math. Gen. 19, L1009 (1986).

22. R.G. Petschek, P. Pfeuty, Phys. Rev. Lett. 58, 1096 (1987).

23. A. Semenov, A. Johner, Eur. Phys. J. E 12, 469 (2003).

24. J. Reiter, G. Zifferer, O.F. Olaj, Macromolecules 22, 3120 (1989).

25. I. Carmesin, K. Kremer, J. Phys. (Paris) 51, 915 (1990).

26. P.H. Nelson, T.A. Hatton, G.C. Rutledge, J. Chem. Phys. 107, 1269 (1997).

27. A. Cavallo, M. Müller, K. Binder, Europhys. Lett. 61, 214 (2003).

28. A. Yethiraj, Macromolecules 36, 5854 (2003).

29. W.A. Kuhlman, E.A. Olivetti, L.G. Griffith, A.M. Mayes, Macromolecules 39, 5122 (2006).

30. H. Aoki, M. Anryu, S. Ito, Polymer 46, 5896 (2005).

31. F.C. Sun, A.V. Dobrynin, D. Shirvanyants, H.-I. Lee, K. Matyjaszewski, G.J. Rubinstein, M. Rubinstein, S.S. Sheiko, Phys. Rev. Lett. 99, 137801 (2007).

32. S.S. Sheiko, B.S. Sumerlin, K. Matyjaszewski, Progr. Polym. Sci. 33, 759 (2008).

33. X. Wang, V.J. Foltz, J. Chem. Phys. 121, 8158 (2004).

34. M.O. Gallyamov, B. Tartsch, A.R. Khokhlov, S.S. Sheiko, H.G. Börner, K. Matyjaszewski, M. Möller, Chem. Eur. J. 10, 4599 (2004).

35. M.O. Gallyamov, B. Tartsch, A.R. Khokhlov, S.S. Sheiko, H.G. Börner, K. Matyjaszewski, M. Möller, J. Microsc. 215, 245 (2004).

36. H.G. Börner, K. Beers, K. Matyjaszewski, S.S. Sheiko, M. Möller, Macromolecules 34, 4375 (2001).

37. T. Pakula, Y. Zhang, K. Matyjaszewski, H.-i. Lee, H. Boerner, S. Qin, G.C. Berry, Polymer 47, 7198 (2006). 
38. M.O. Gallyamov, A.R. Khokhlov, M. Möller, Macromol. Rapid Commun. 26, 456 (2005).

39. M.O. Gallyamov, B. Tartsch, P. Mela, H. Börner, K. Matyjaszewski, S. Sheiko, A. Khokhlov, M. Möller, Phys. Chem. Chem. Phys. 9, 346 (2007).

40. S.S. Sheiko, F.C. Sun, A. Randall, D. Shirvanyants, M. Rubinstein, H.-i. Lee, K. Matyjaszewski, Nature 440, 191 (2006).

41. I.I. Potemkin, A.R. Khokhlov, S. Prokhorova, S.S. Sheiko,
M. Möller, K.L. Beers, K. Matyjaszewski, Macromolecules 37, 3918 (2004).

42. I.I. Potemkin, Eur. Phys. J. E 12, 207 (2003).

43. I.I. Potemkin, K.I. Popov, J. Chem. Phys. 129, 124901 (2008).

44. I.I. Potemkin, A.R. Khokhlov, P. Reineker, Eur. Phys. J. E 4, 93 (2001).

45. I.I. Potemkin, Macromolecules 39, 7178 (2006).

46. I.I. Potemkin, M. Möller, Macromolecules 38, 2999 (2005). 